\title{
Hard loop approach to anisotropic systems
}

\author{
Stanisław Mrówczyński* \\ Sottan Institute for Nuclear Studies ul. Hoża 69, PL - 00-681 Warsaw, Poland \\ and Institute of Physics, Pedagogical University ul. Konopnickiej 15, PL - 25-406 Kielce, Poland \\ Markus H. Thoma ${ }^{\dagger}$ \\ Theory Division, CERN, CH-1211 Geneva 23, Switzerland \\ (Received 18 January 2000; published 12 July 2000)
}

\begin{abstract}
Anisotropic systems of quarks and gluons, which at least for sufficiently short space-time intervals can be treated as homogeneous and static, are considered. The gluon polarization tensor of such a system is explicitly computed within the semiclassical kinetic and hard loop diagrammatic theories. The equivalence of the two approaches is demonstrated. The quark self-energy is computed as well, and finally, the dispersion relations of quarks and gluons in the anisotropic medium are discussed.

PACS number(s): 11.10.Wx, 05.20.Dd, 12.38.Mh
\end{abstract}

\section{INTRODUCTION}

The state of equilibrium, being static and homogeneous, is sometimes anisotropic. This may happen when the system of quantum fields, which is of interest here, is under the influence of an external force. A relativistic plasma, which is anisotropic due to a magnetic field, often occurs in astrophysical situations such as the early Universe or supernovas [1]. Anisotropic states are also common for systems which are out of equilibrium. Sometimes such states can be treated as static and homogeneous, but only for sufficiently short time and space intervals. How short the intervals should be depends on the specific problem under consideration.

The parton system generated at the early stage of ultrarelativistic heavy ion collisions at the BNL Relativistic Heavy Ion Collider (RHC) or CERN Large Hadon Collider (LHC) is of particular interest for us. The parton momentum distribution is not isotropic but strongly elongated along the beam $[2,3]$. Therefore, specific color fluctuations, instead of being damped, can exponentially grow and noticeably influence the temporal evolution of the system. In a series of papers by one of us [4,5], it has been argued that there are indeed very fast unstable plasma modes in such a parton system. The stability analysis [4,5] has been performed within the semiclassical transport theory of quarks and gluons $[6,7]$. Since the theory has been proven till now to be fully consistent with the QCD dynamics only for quasiequilibrium systems $[8,9]$ one wonders to what extent the results from $[4,5]$ are reliable. Thus, a QCD diagrammatic analysis is desirable.

Perturbative approaches within the real time field theory provide a natural framework to study weakly interacting quantum field systems in and out of equilibrium. However, the naive perturbative expansion, when applied to gauge fields, suffers from various singularities and some physical quantities are even gauge dependent. These problems have been partly resolved for equilibrium systems by using an

\footnotetext{
*Electronic address: mrow@fuw.edu.pl

†Electronic address: markus.thoma@cern.ch
}

effective perturbative expansion where the so-called hard thermal loops are resummed [10]. The hard thermal loop resummation technique within finite-temperature QCD has been shown to be equivalent to the approach based on the classical [11] transport equations, where color is treated as a classical variable, or on the semiclassical [8] one, where the color degrees of freedom emerge due to the matrix structure of the parton distribution function. The hard thermal loop approach has been generalized to nonequilibrium systems, but only very specific forms of deviations from the equilibrium have been discussed so far: systems out of chemical equilibrium, which are important in the context of heavy-ion collisions [12], and such where the momentum distribution is isotropic but not of the Bose-Einstein or Fermi-Dirac form [13-15]. As observed in [16], the hard thermal loop approach can be applied to any momentum distribution of hard particles which is static and homogeneous. This is evident when the hard thermal loop effective action is derived within the transport theory [16]. The term "thermal" is then rather misleading and for this reason we omit it in the following.

In this paper we discuss the applicability of the hard loop technique for systems with anisotropic momentum distributions. The technique has been earlier applied to the equilibrium QED plasma in a magnetic field [17]. Our aim is to consider a general situation with an arbitrary momentum distribution. We analyze the problem from the point of view of the transport theory and the diagrammatic approach. Using the semiclassical kinetic equations we derive the hard-loopinduced current paying much attention to the gauge aspects of the procedure. We also explicitly demonstrate that the gluon polarization tensors found by means of the two approaches are identical. In this way, the applicability of the kinetic theory beyond the equilibrium is substantiated and more specifically, the reliability of the results from $[4,5]$ is shown.

The hard loop diagrammatic technique has the advantage over the semiclassical transport theory approach that it can be naturally extended to fermionic self-energies and to higher-order diagrams beyond the semiclassical approximation. In this way the dispersion relations of quarks and other observables of the quark-gluon plasma, such as the energy 
loss of energetic partons, transport coefficients, or photon and dilepton production rates [18], can be calculated systematically in the case of anisotropic distributions. We take a first step in this direction computing the quark self-energy for an arbitrary momentum distribution.

The self-energy controls the particle dispersion relation which provides an essential dynamical information about the system. We discuss therefore the general dispersion relation of gluons (plasmons) and quarks in the anisotropic quarkgluon plasma. Finally, we briefly consider possible applications of the formalism developed in this paper.

\section{TRANSPORT THEORY APPROACH}

In this section we first introduce the semiclassical transport theory of quarks and gluons [6,7]. Then, applying the linear-response method, the hard-loop-induced current is derived. Finally, we compute the gluon polarization tensor.

\section{A. Transport equations}

The distribution function of hard (anti-)quarks $Q(\mathbf{p}, x)$ $[\bar{Q}(\mathbf{p}, x)]$ is a Hermitian $N_{c} \times N_{c}$ matrix in color space [for a $S U\left(N_{c}\right)$ color group]; $x$ denotes the space-time quark coordinate and $\mathbf{p}$ is its momentum. The four-momentum $p$ $=(E, \mathbf{p})$ is assumed to satisfy the mass-shell constraint. Since both quarks and gluons are treated as massless particles the constraint is $p^{2}=0$. We also mention here that the spin of quarks and gluons is taken into account as an internal degree of freedom. The distribution function transforms under local gauge transformation $M$ as

$$
Q(\mathbf{p}, x) \rightarrow M(x) Q(\mathbf{p}, x) M^{\dagger}(x) .
$$

The color indices are here and in the most cases below suppressed. The distribution function of hard gluons is a Hermitian $\left(N_{c}^{2}-1\right) \times\left(N_{c}^{2}-1\right)$ matrix which transforms as

$$
G(\mathbf{p}, x) \rightarrow \mathcal{M}(x) G(\mathbf{p}, x) \mathcal{M}^{\dagger}(x),
$$

where

$$
\mathcal{M}_{a b}(x)=\operatorname{Tr}\left[\tau_{a} M(x) \tau_{b} M^{\dagger}(x)\right]
$$

with $\tau_{a}, a=1, \ldots, N_{c}^{2}-1$ being the $\mathrm{SU}\left(N_{c}\right)$ group generators in the fundamental representation.

The color current is expressed in the fundamental representation as

$$
\begin{aligned}
j^{\mu}(x)= & -g \int_{\frac{d^{3} p}{(2 \pi)^{3} 2 E} p^{\mu}[Q(\mathbf{p}, x)-\bar{Q}(\mathbf{p}, x)} \\
& \left.-\frac{1}{N_{c}} \operatorname{Tr}[Q(\mathbf{p}, x)-\bar{Q}(\mathbf{p}, x)]+2 i \tau_{a} f_{a b c} G_{b c}(\mathbf{p}, x)\right],
\end{aligned}
$$

where $g$ is the QCD coupling constant, $f_{a b c}$ are the structure constants of the $S U\left(N_{c}\right)$ group.

The distribution functions of quarks and gluons are assumed to satisfy the following collisionless transport equations:

$$
\begin{aligned}
& p^{\mu} D_{\mu} Q(\mathbf{p}, x)+g p^{\mu} \frac{\partial}{\partial p_{\nu}} \frac{1}{2}\left\{F_{\mu \nu}(x), Q(\mathbf{p}, x)\right\}=0, \\
& p^{\mu} D_{\mu} \bar{Q}(\mathbf{p}, x)-g p^{\mu} \frac{\partial}{\partial p_{\nu}} \frac{1}{2}\left\{F_{\mu \nu}(x), \bar{Q}(\mathbf{p}, x)\right\}=0, \\
& p^{\mu} \mathcal{D}_{\mu} G(\mathbf{p}, x)+g p^{\mu} \frac{\partial}{\partial p_{\nu}} \frac{1}{2}\left\{\mathcal{F}_{\mu \nu}(x), G(\mathbf{p}, x)\right\}=0,
\end{aligned}
$$

where $\{\ldots, \ldots\}$ denotes the anicommutator; $D_{\mu}$ and $\mathcal{D}_{\mu}$ are the covariant derivatives which act as

$$
D_{\mu}=\partial_{\mu}-i g\left[A_{\mu}(x), \ldots\right] \quad \mathcal{D}_{\mu}=\partial_{\mu}-i g\left[\mathcal{A}_{\mu}(x), \ldots\right],
$$

with $A_{\mu}$ and $\mathcal{A}_{\mu}$ being the mean-field or background four potentials;

$$
A^{\mu}(x)=A_{a}^{\mu}(x) \tau_{a}, \quad \mathcal{A}_{a b}^{\mu}(x)=-i f_{a b c} A_{c}^{\mu}(x) ;
$$

$F_{\mu \nu}$ and $\mathcal{F}_{\mu \nu}$ are the mean-field stress tensors with a color index structure analogous to that of the four potentials. The background field is generated by the color current (3) and the respective equation is

$$
D_{\mu} F^{\mu \nu}(x)=j^{\nu}(x) .
$$

We note that the set of transport equations (4), (5) is covariant with respect to the gauge transformations (1), (2).

\section{B. Plasma color response}

We discuss here how the plasma, which is (on average) colorless, homogeneous, and stationary, responds to small color fluctuations. The distribution functions are assumed to be of the form

$$
\begin{gathered}
Q_{i j}(\mathbf{p}, x)=n(\mathbf{p}) \delta_{i j}+\delta Q_{i j}(\mathbf{p}, x), \\
\bar{Q}_{i j}(\mathbf{p}, x)=\bar{n}(\mathbf{p}) \delta_{i j}+\delta \bar{Q}_{i j}(\mathbf{p}, x), \\
G_{a b}(\mathbf{p}, x)=n_{g}(\mathbf{p}) \delta_{a b}+\delta G_{a b}(\mathbf{p}, x),
\end{gathered}
$$

where the functions describing the deviation from the colorless state are assumed to be much smaller than the respective colorless functions. The same is assumed for the momentum gradients of these functions. The (anti-)quark and gluon distribution functions $n(\mathbf{p}), \bar{n}(\mathbf{p}), n_{g}(\mathbf{p})$, reduce in equilibrium to the Fermi-Dirac or Bose-Einstein form, i.e.,

$$
\begin{gathered}
n(\mathbf{p})=\frac{2}{\exp (|\mathbf{p}|-\mu) / T+1}, \\
\bar{n}(\mathbf{p})=\frac{2}{\exp (|\mathbf{p}|+\mu) / T+1}, \\
\mathrm{n}_{\mathrm{g}}(\mathbf{p})=\frac{2}{\exp (|\mathbf{p}| / T)-1},
\end{gathered}
$$

where $T$ and $\mu$ denote the temperature and quark chemical potential, respectively, while the factor of 2 occurs due to the spin degrees of freedom. The number of quark flavors is assumed to be equal to one.

Substituting Eq. (6) in Eq. (3) one gets 


$$
\begin{aligned}
j^{\mu}(x)= & -g \int_{0} \frac{d^{3} p}{(2 \pi)^{3} 2 E} p^{\mu}[\delta Q(\mathbf{p}, x)-\delta \bar{Q}(\mathbf{p}, x) \\
& -\frac{1}{N_{c}} \operatorname{Tr}[\delta Q(\mathbf{p}, x)-\delta \bar{Q}(\mathbf{p}, x)] \\
& \left.+2 i \tau_{a} f_{a b c} \delta G_{b c}(\mathbf{p}, x)\right]
\end{aligned}
$$

As seen, the current occurs due to the deviation from the colorless state. Let us also observe here that not only (anti-)quarks but also gluons contribute to the current (8). Thus, the current is of essentially a non-Abelian nature.

Now, we substitute the distribution functions (6) into the transport equations (4). Assuming that the stress tensor is of the same order as $\delta Q, \delta \bar{Q}$ or $\delta G$ and linearizing the equations with respect to $\delta Q, \delta \bar{Q}$, and $\delta G$ we get

$$
\begin{aligned}
& p^{\mu} D_{\mu} \delta Q(\mathbf{p}, x)=-g p^{\mu} F_{\mu \nu}(x) \frac{\partial n(\mathbf{p})}{\partial p_{\nu}} \\
& p^{\mu} D_{\mu} \delta \bar{Q}(\mathbf{p}, x)=g p^{\mu} F_{\mu \nu}(x) \frac{\partial \bar{n}(\mathbf{p})}{\partial p_{\nu}}, \\
& p^{\mu} \mathcal{D}_{\mu} \delta G(\mathbf{p}, x)=-g p^{\mu} \mathcal{F}_{\mu \nu}(x) \frac{\partial n_{g}(\mathbf{p})}{\partial p_{\nu}} .
\end{aligned}
$$

We keep here the covariant derivatives to maintain the gauge covariance of the equations.

To solve the equations such as Eqs. (9) one usually uses, see, e.g., $[6,8]$, the gauge parallel transporter defined in the fundamental representation as

$$
U(x, y)=\mathcal{P} \exp \left[-i g \int_{x}^{y} d z_{\mu} A^{\mu}(z)\right]
$$

where $\mathcal{P}$ denotes the ordering along the path from $x$ to $y$. There is an analogous formula of the gauge transporter $\mathcal{U}(x, y)$ in the adjoint representation. Using $U$ and $\mathcal{U}$ one finds the solutions of Eqs. (9) as

$$
\begin{aligned}
\delta Q(\mathbf{p}, x)= & -g \int d^{4} y G_{p}(x-y) \\
& \times U(x, y) p^{\mu} F_{\mu \nu}(y) U(y, x) \frac{\partial n(\mathbf{p})}{\partial p_{\nu}}, \\
\delta \bar{Q}(\mathbf{p}, x)= & g \int d^{4} y G_{p}(x-y) \\
& \times U(x, y) p^{\mu} F_{\mu \nu}(y) U(y, x) \frac{\partial \bar{n}(\mathbf{p})}{\partial p_{\nu}}, \\
\delta G(\mathbf{p}, x)= & -g \int d^{4} y G_{p}(x-y) \\
& \times \mathcal{U}(x, y) p^{\mu} \mathcal{F}_{\mu \nu}(y) \mathcal{U}(y, x) \frac{\partial n_{g}(\mathbf{p})}{\partial p_{\nu}},
\end{aligned}
$$

where $G_{p}(x)$ is the retarded Green's function which satisfies the equation

$$
p_{\mu} \partial^{\mu} G_{p}(x)=\delta^{(4)}(x)
$$

and equals

$$
G_{p}(x)=E^{-1} \Theta(t) \delta^{(3)}(\mathbf{x}-\mathbf{v} t),
$$

with $t$ being the 0 th component of $x\left[x^{\mu} \equiv(t, \mathbf{x})\right]$, and $\mathbf{v}$ denoting the parton velocity, i.e., $\mathbf{v} \equiv \mathbf{p} / E$.

Substituting the solutions (10) in Eq. (8) one finds the color current of the gauge covariant form which reads

$$
\begin{aligned}
j^{\mu}(x)= & g^{2} \int \frac{d^{3} p}{(2 \pi)^{3} 2 E} p^{\mu} p^{\lambda} \\
& \times \int d^{4} y G_{p}(x-y) U(x, y) F_{\lambda \nu}(y) U(y, x) \frac{\partial f(\mathbf{p})}{\partial p_{\nu}},
\end{aligned}
$$

where $f(\mathbf{p}) \equiv n(\mathbf{p})+\bar{n}(\mathbf{p})+2 N_{c} n_{g}(\mathbf{p})$.

Now, we are going to perform the Fourier transform of the induced current (11). Before this step however, we neglect the terms which are not of the leading order in $g$. Then, the transporters $U$ are approximated by unity and the stress tensor $F_{\mu \nu}$ by $\partial_{\mu} A_{\nu}-\partial_{\nu} A_{\mu}$. Within such an approximation, the Fourier-transform-induced current (11), which is no longer gauge covariant, equals

$$
j^{\mu}(k)=g^{2} \int \frac{d^{3} p}{(2 \pi)^{3} 2 E} p^{\mu} \frac{\partial f(\mathbf{p})}{\partial p_{\lambda}}\left[g^{\lambda \nu}-\frac{k^{\lambda} p^{\nu}}{p^{\sigma} k_{\sigma}+i 0^{+}}\right] A_{\nu}(k) .
$$

The induced current $j^{\mu}(k)$ can be expressed as

$$
j_{a}^{\mu}(k)=\Pi_{a b}^{\mu \nu}(k) A_{\nu}^{b}(k),
$$

with $\Pi^{\mu \nu}$ being the gluon polarization tensor. Transforming Eq. (12) to the adjoint representation one finds

$$
\Pi^{\mu \nu}(k)=g^{2} \int \frac{d^{3} p}{(2 \pi)^{3} 2 E} p^{\mu} \frac{\partial f(\mathbf{p})}{\partial p_{\lambda}}\left[g^{\lambda \nu}-\frac{k^{\lambda} p^{\nu}}{p^{\sigma} k_{\sigma}+i 0^{+}}\right] .
$$

It should be noted here that the polarization tensor is proportional to a unit matrix in the color space.

Now we are going to show that the polarization tensor is transversal, i.e., $k_{\mu} \Pi^{\mu \nu}(k)=0$. Let us first consider $k_{\mu} \Pi^{\mu 0}(k)$. One immediately finds from Eq. (13) that

$$
k_{\mu} \Pi^{\mu 0}(k)=-\frac{g^{2}}{2} k^{l} \int \frac{d^{3} p}{(2 \pi)^{3}} \frac{\partial f(\mathbf{p})}{\partial p^{l}} .
$$

The indices $l, m, n=1,2,3$ refer to the coordinates of three vectors. The energy density carried by partons is expected to be finite. Therefore, $f(\mathbf{p}=\infty)$ must vanish. Consequently, the above integral vanishes as well. Performing a partial integration and demanding that $f(\mathbf{p}=\infty)=0$, one also proves that $k_{\mu} \Pi^{\mu m}(k)=0$. Analogously, it can be also shown that $\Pi^{\mu \nu}(k)=\Pi^{\nu \mu}(k)$.

\section{DIAGRAMMATIC APPROACH}

In this section we consider the diagrammatic hard loop approach to anisotropic systems. Specifically, we compute 
the QCD polarization tensor and the quark self-energy for an arbitrary parton momentum distribution.

\section{A. Polarization tensor}

The contribution from the quark loop to the gluon selfenergy in the case of one quark flavor is of the form

$$
\Pi_{a b}^{\mu \nu}(k)=\frac{i}{2} \delta_{a b} g^{2} \int \frac{d^{4} p}{(2 \pi)^{4}} \operatorname{Tr}\left[\gamma^{\mu} S(q) \gamma^{\nu} S(p)\right]
$$

where $q \equiv p-k$ and $S$ is the bare quark propagator. Since we are dealing with a non-equilibrium situation we adopt the real time formalism. Within the Keldysh representation [19], which has been shown to be especially convenient in the hard loop approximation [15], there are retarded $(R)$, advanced $(A)$, and symmetric $(F)$ propagators which in the case of massless quarks are given by

$$
\begin{aligned}
S_{R, A}(p)= & \frac{p}{p^{2} \pm i \operatorname{sgn}\left(p_{0}\right) 0^{+}}, \\
S_{F}(p)= & -2 \pi i p\left\{\left\{[1-n(\mathbf{p})] \Theta\left(p_{0}\right)\right.\right. \\
& \left.+[1-\bar{n}(\mathbf{p})] \Theta\left(-p_{0}\right)\right\} \delta\left(p^{2}\right),
\end{aligned}
$$

where $n(\mathbf{p})(\bar{n}(\mathbf{p}))$ is, as previously, the (anti-)quark distribution function [14] that reduces in equilibrium to the form (7). Performing the trace in Eq. (14) and suppressing the color indices, we find the retarded gluon self-energy as

$$
\begin{aligned}
\Pi^{\mu \nu}(k)= & i g^{2} \int \frac{d^{4} p}{(2 \pi)^{4}}\left[q^{\mu} p^{\nu}+p^{\mu} q^{\nu}-g^{\mu \nu}(q \cdot p)\right] \\
& \times\left[\tilde{\Delta}_{F}(q) \tilde{\Delta}_{R}(p)+\widetilde{\Delta}_{A}(q) \tilde{\Delta}_{F}(p)\right],
\end{aligned}
$$

where $\quad S_{R, A, F}(p)=p p \widetilde{\Delta}_{R, A, F}(p)$. Terms containing $\widetilde{\Delta}_{A}(q) \widetilde{\Delta}_{A}(p)$ and $\widetilde{\Delta}_{R}(q) \widetilde{\Delta}_{R}(p)$ have been neglected as they vanish after integrating over $p_{0}$.

First, we will consider the spatial components of the polarization tensor. The other components follow from it using the transversality of the polarization tensor, as we will discuss below. After performing the integration over $p_{0}$, we obtain

$$
\Pi^{l m}(k)=-\frac{g^{2}}{2} \int \frac{d^{3} p}{(2 \pi)^{3}} \frac{f_{q}(\mathbf{p})}{|\mathbf{p}|}\left[\frac{2 p^{l} p^{m}-k^{l} p^{m}-p^{l} k^{m}+\delta^{l m}(-\omega|\mathbf{p}|+\mathbf{k} \cdot \mathbf{p})}{-2 \omega|\mathbf{p}|+2 \mathbf{k} \cdot \mathbf{p}+k^{2}-i \operatorname{sgn}(|\mathbf{p}|-\omega) 0^{+}}+\frac{2 p^{l} p^{m}-k^{l} p^{m}-p^{l} k^{m}+\delta^{l m}(\omega|\mathbf{p}|+\mathbf{k} \cdot \mathbf{p})}{2 \omega|\mathbf{p}|+2 \mathbf{k} \cdot \mathbf{p}+k^{2}-i \operatorname{sgn}(-|\mathbf{p}|-\omega) 0^{+}}\right]
$$

where $\omega$ is the 0 th component of $k$, i.e., $k=(\omega, \mathbf{k})$ and $f_{q}(\mathbf{p}) \equiv n(\mathbf{p})+\bar{n}(\mathbf{p})$. Here the vacuum part has been neglected because it is suppressed compared to the matter part in the hard loop approximation.

Adopting the hard loop approximation we assume that the internal momenta are much larger than the external one, i.e., $\omega$, $k_{l} \ll p_{l}$. Note that for arbitrary anisotropic distributions we have to require the hard loop condition for each component of the momenta, whereas in the isotropic case $\omega,|\mathbf{k}| \ll|\mathbf{p}|$ suffices. Expanding the expression in the square brackets for small external momenta yields

$$
\begin{aligned}
& \frac{p^{l} p^{m}}{-\omega|\mathbf{p}|+\mathbf{k} \cdot \mathbf{p}-i 0^{+}}+\frac{p^{l} p^{m}}{\omega|\mathbf{p}|+\mathbf{k} \cdot \mathbf{p}+i 0^{+}}+\frac{-k^{l} p^{m}-p^{l} k^{m}+\delta^{l m}(-\omega|\mathbf{p}|+\mathbf{k} \cdot \mathbf{p})}{2\left(-\omega|\mathbf{p}|+\mathbf{k} \cdot \mathbf{p}-i 0^{+}\right)}+\frac{-k^{l} p^{m}-p^{l} k^{m}+\delta^{l m}(\omega|\mathbf{p}|+\mathbf{k} \cdot \mathbf{p})}{2\left(\omega|\mathbf{p}|+\mathbf{k} \cdot \mathbf{p}+i 0^{+}\right)} \\
& -\frac{p^{l} p^{m} k^{2}}{2\left(-\omega|\mathbf{p}|+\mathbf{k} \cdot \mathbf{p}-i 0^{+}\right)^{2}}-\frac{p^{l} p^{m} k^{2}}{2\left(\omega|\mathbf{p}|+\mathbf{k} \cdot \mathbf{p}+i 0^{+}\right)^{2}}
\end{aligned}
$$

In equilibrium the first two terms vanish after integrating over $\mathbf{p}$. This also holds out of equilibrium if we assume $f_{q}(-\mathbf{p})$ $=f_{q}(\mathbf{p})$. Then, we arrive at the final result

$$
\Pi^{l m}(k)=-\frac{g^{2}}{2} \int \frac{d^{3} p}{(2 \pi)^{3}} \frac{f(\mathbf{p})}{|\mathbf{p}|} \frac{\left(k^{l} p^{m}+p^{l} k^{m}\right)(\omega|\mathbf{p}|-\mathbf{k} \cdot \mathbf{p})+\delta^{l m}(\omega|\mathbf{p}|-\mathbf{k} \cdot \mathbf{p})^{2}-p^{l} p^{m}\left(\omega^{2}-|\mathbf{k}|^{2}\right)}{\left(\omega|\mathbf{p}|-\mathbf{k} \cdot \mathbf{p}+i 0^{+}\right)^{2}}
$$


where we replaced $f_{q}(\mathbf{p})$ by $f(\mathbf{p}) \equiv n(\mathbf{p})+\bar{n}(\mathbf{p})+2 N_{c} n_{g}(\mathbf{p})$. The point is that in the hard loop limit the gluonic contributions to the polarization tensor have the same structure as the quark ones [20] and only the distribution function and the color factor change. For essentially the same reason, the QCD polarization tensor, computed even with the complete expression (18) without assuming $f(-\mathbf{p})=f(\mathbf{p})$, is gauge independent in the hard loop approximation. Indeed, the gluon polarization tensor has the same structure as the photon one in the hard loop limit. Since the one-loop photon polarization tensor contains no gauge boson propagator it is gauge independent. Consequently, the same holds for the gluon polarization tensor. The result (19) is fully equivalent to Eq. (13) obtained within the semiclassical kinetic theory. In order to show the equivalence, one performs a partial integration in Eq. (13) and immediately gets Eq. (19). However, we do not need to assume the reflection symmetry of the distribution function to derive Eq. (13).

Two more comments are in order here. First, if we do not assume the reflection symmetry of $f(\mathbf{p})$ the first two terms in Eq. (18) will contribute, leading to contributions in the polarization tensor that dominate over the hard loop result (19) and are absent in the semiclassical approximation. As explained above, the extra contribution appears to be gauge independent. Second, for equilibrium distribution functions (7) the integrals over $|\mathbf{p}|$ and over the angle in Eq. (19) factorize. Then, it is easy to show that Eq. (19) reduces to the well-known hard thermal loop result [21] where the polarization tensor has only two independent components and depends on $\omega$ and $|\mathbf{k}|$.

Owing to transversality, the timelike components of $\Pi^{\mu \nu}$ follow from $\Pi^{l m}$. Indeed, $\Pi^{0 m}(k)=k^{l} \Pi^{l m}(k) / \omega$ and $\Pi^{00}(k)=k^{l} k^{m} \Pi^{l m}(k) / \omega^{2}$. In order to prove the transversality of the hard loop polarization tensor in the case of anisotropic distributions, we compute $k_{\mu} \Pi^{\mu \nu}(k)$. Considering first the quark loop contribution, we get

$$
\begin{aligned}
k_{\mu} \Pi^{\mu \nu}(k)= & i g^{2} \int \frac{d^{4} p}{(2 \pi)^{4}}\left[2(k \cdot p) p^{\nu}-p^{2} k^{\nu}-k^{2} p^{\nu}\right] \\
& \times\left[\widetilde{\Delta}_{F}(q) \widetilde{\Delta}_{R}(p)+\widetilde{\Delta}_{A}(q) \widetilde{\Delta}_{F}(p)\right] .
\end{aligned}
$$

After integrating over $p_{0}$ we find

$$
k_{\mu} \Pi^{\mu 0}(k)=-\frac{g^{2}}{2} \int \frac{d^{3} p}{(2 \pi)^{3}} \frac{f_{q}(\mathbf{p})}{|\mathbf{p}|}\left[\frac{2(\omega|\mathbf{p}|-\mathbf{k} \cdot \mathbf{p})|\mathbf{p}|-k^{2}|\mathbf{p}|}{-2 \omega|\mathbf{p}|+2 \mathbf{k} \cdot \mathbf{p}+k^{2}-i \operatorname{sgn}(|\mathbf{p}|-\omega) 0^{+}}+\frac{-2(-\omega|\mathbf{p}|-\mathbf{k} \cdot \mathbf{p})|\mathbf{p}|+k^{2}|\mathbf{p}|}{2 \omega|\mathbf{p}|+2 \mathbf{k} \cdot \mathbf{p}+k^{2}-i \operatorname{sgn}(-|\mathbf{p}|-\omega) 0^{+}}\right]
$$

and

$$
k_{\mu} \Pi^{\mu m}(k)=-\frac{g^{2}}{2} \int \frac{d^{3} p}{(2 \pi)^{3}} \frac{f_{q}(\mathbf{p})}{|\mathbf{p}|}\left[\frac{2(\omega|\mathbf{p}|-\mathbf{k} \cdot \mathbf{p}) p^{m}-k^{2} p^{m}}{-2 \omega|\mathbf{p}|+2 \mathbf{k} \cdot \mathbf{p}+k^{2}-i \operatorname{sgn}(|\mathbf{p}|-\omega) 0^{+}}+\frac{2(-\omega|\mathbf{p}|-\mathbf{k} \cdot \mathbf{p}) p^{m}-k^{2} p^{m}}{2 \omega|\mathbf{p}|+2 \mathbf{k} \cdot \mathbf{p}+k^{2}-i \operatorname{sgn}(-|\mathbf{p}|-\omega) 0^{+}}\right] .
$$

Expanding the integrands in these expressions for small external momenta analogously to Eq. (18), it is easy to show that Eqs. (21) and (22) vanish in the hard loop approximation. This also holds if the gluon loop contribution is added as they have the same structure in the hard loop approximation.

\section{B. Fermion self-energy}

As mentioned in the Introduction, the diagrammatic technique has the advantage over the semiclassical transport theory approach that it can be easily extended to fermionic self-energies. Therefore, we discuss the hard loop quark selfenergy for anisotropic momentum distributions. Using the Feynman gauge, the one-loop quark self-energy is found as

$$
\Sigma_{i j}(k)=2 i C_{F} \delta_{i j} g^{2} \int \frac{d^{4} p}{(2 \pi)^{4}} S(p) \Delta(q)
$$

where $C_{F} \equiv\left(N_{c}^{2}-1\right) / N_{c}$ and now $q \equiv k-p$. Adopting again the Keldysh representation, the gluon propagators in the Feynman gauge are given by

$$
\begin{aligned}
\Delta_{R, A}(q) & =\frac{1}{q^{2} \pm i \operatorname{sgn}\left(q_{0}\right) 0^{+}}, \\
\Delta_{F}(q) & =-2 \pi i\left[1+n_{g}(\mathbf{q})\right] \delta\left(q^{2}\right) .
\end{aligned}
$$

Suppressing the color indces, we find for the retarded quark self-energy

$$
\begin{aligned}
\Sigma(k)= & i g^{2} C_{F} \int \frac{d^{4} p}{(2 \pi)^{4}} p\left[\widetilde{\Delta}_{R}(p) \Delta_{F}(q)+\widetilde{\Delta}_{R}(p) \Delta_{A}(q)\right. \\
& \left.+\widetilde{\Delta}_{F}(p) \Delta_{R}(q)+\widetilde{\Delta}_{A}(p) \Delta_{R}(q)\right] .
\end{aligned}
$$

The matter part of Eq. (25) can be decomposed into two contributions which read after integrating over $p_{0}$ 


$$
\Sigma_{1}(k)=\frac{g^{2}}{8} C_{F} \int \frac{d^{3} p}{(2 \pi)^{3}} \frac{n_{g}(\mathbf{p})}{|\mathbf{p}|}\left[\frac{(\omega-p) \gamma_{0}-(\mathbf{k}-\mathbf{p}) \cdot \boldsymbol{\gamma}}{-2 \omega|\mathbf{p}|+2 \mathbf{k} \cdot \mathbf{p}+k^{2}+i \operatorname{sgn}(\omega-|\mathbf{p}|) 0^{+}}+\frac{(\omega+p) \gamma_{0}-(\mathbf{k}-\mathbf{p}) \cdot \boldsymbol{\gamma}}{2 \omega|\mathbf{p}|+2 \mathbf{k} \cdot \mathbf{p}+k^{2}+i \operatorname{sgn}(\omega+|\mathbf{p}|) 0^{+}}\right]
$$

and

$$
\Sigma_{2}(k)=\frac{g^{2}}{16} C_{F} \int \frac{d^{3} p}{(2 \pi)^{3}} \frac{n(\mathbf{p})+\bar{n}(\mathbf{p})}{|\mathbf{p}|}\left[\frac{-p \gamma_{0}+\mathbf{p} \cdot \boldsymbol{\gamma}}{-2 \omega|\mathbf{p}|+2 \mathbf{k} \cdot \mathbf{p}+k^{2}+i \operatorname{sgn}(\omega-|\mathbf{p}|) 0^{+}}+\frac{p \gamma_{0}+\mathbf{p} \cdot \boldsymbol{\gamma}}{2 \omega|\mathbf{p}|+2 \mathbf{k} \cdot \mathbf{p}+k^{2}+i \operatorname{sgn}(\omega+|\mathbf{p}|) 0^{+}}\right] .
$$

In contrast to the polarization tensor, we need to expand the square brackets in Eq. (26) only to the first order for small external momenta, leading to

$$
\frac{-p \gamma_{0}+\mathbf{p} \cdot \boldsymbol{\gamma}}{-2 \omega|\mathbf{p}|+2 \mathbf{k} \cdot \mathbf{p}-i 0^{+}}+\frac{p \gamma_{0}+\mathbf{p} \cdot \boldsymbol{\gamma}}{2 \omega|\mathbf{p}|+2 \mathbf{k} \cdot \mathbf{p}+i 0^{+}}
$$

Assuming again the reflection symmetry for the distribution functions, we obtain the final gauge-independent result in the hard loop approximation

$$
\begin{aligned}
\Sigma(k)= & \frac{g^{2}}{16} C_{F} \int \frac{d^{3} p}{(2 \pi)^{3}} \frac{2 n_{g}(\mathbf{p})+n(\mathbf{p})+\bar{n}(\mathbf{p})}{|\mathbf{p}|} \\
& \times \frac{\gamma_{0}+\mathbf{v} \cdot \boldsymbol{\gamma}}{\omega+\mathbf{v} \cdot \mathbf{k}+i 0^{+}} .
\end{aligned}
$$

In the case of isotropic distributions (29) reduces to the wellknown hard thermal loop result [22], where the self-energy for massless quarks contains only two independent scalar functions depending on $\omega$ and $|\mathbf{k}|$. Giving up the reflection symmetry of the distribution functions does not introduce new dominant terms in this case since the self-energy follows already from the lowest order terms (28). We have adopted this symmetry to treat $\Pi$ and $\Sigma$ in exactly the same way.

\section{DISPERSION RELATIONS}

The gluon polarization tensor and quark self-energy can be used to determine the dispersion relations of gluons (plasmons) and quarks in the quasistatic and quasihomogeneous but anisotropic state of the quark-gluon plasma.

\section{A. Gluon dispersion equation}

The background gluon field $A^{\mu}(k)$ satisfies the following equation of motion:

$$
\left[k^{2} g^{\mu \nu}-k^{\mu} k^{\nu}-\Pi^{\mu \nu}(k)\right] A_{\nu}(k)=0 .
$$

Therefore, the general plasmon dispersion equation is of the form

$$
\operatorname{det}\left[k^{2} g^{\mu \nu}-k^{\mu} k^{\nu}-\Pi^{\mu \nu}(k)\right]=0 .
$$

Equivalently, the dispersion relations are given by the positions of the pole of the effective gluon propagator. Due to the transversality of $\Pi^{\mu \nu}$ not all components of $\Pi^{\mu \nu}$ are independent from each other and consequently the dispersion equation (30) can be simplified. For this purpose we introduce the color permittivity tensor $\epsilon^{l m}(k)$. Because of the relation

$$
\epsilon^{l m}(k) E^{l}(k) E^{m}(k)=\Pi^{\mu \nu}(k) A_{\mu}(k) A_{\nu}(k),
$$

where $\mathbf{E}$ is the chromoelectric vector, the permittivity can be expressed through the polarization tensor as

$$
\epsilon^{l m}(k)=\delta^{l m}+\frac{1}{\omega^{2}} \Pi^{l m}(k)
$$

There are two other equalities which follow from the transversality of $\Pi^{\mu \nu}$. Namely,

$$
\begin{aligned}
& \Pi^{00}(k)=\left[\epsilon^{l m}(k)-\delta^{l m}\right] k^{l} k^{m}, \\
& \Pi^{l 0}(k)=\left[\epsilon^{l m}(k)-\delta^{l m}\right] \omega k^{m} .
\end{aligned}
$$

Using the permittivity tensor the dispersion equation gets the form

$$
\operatorname{det}\left[\mathbf{k}^{2} \delta^{l m}-k^{l} k^{m}-\omega^{2} \epsilon^{l m}(k)\right]=0
$$

with

$$
\begin{aligned}
\epsilon^{l m}(k)= & \delta^{l m}+\frac{g^{2}}{2 \omega} \int \frac{d^{3} p}{(2 \pi)^{3}} \frac{v^{l}}{\omega-\mathbf{k} \cdot v+i 0^{+}} \frac{\partial f(\mathbf{p})}{\partial p^{n}} \\
& \times\left[\left(1-\frac{\mathbf{k} \cdot \mathbf{v}}{\omega}\right) \delta^{n m}+\frac{k^{n} v^{m}}{\omega}\right] .
\end{aligned}
$$

In the isotropic state there are only two independent components of the permittivity tensor

$$
\left.\boldsymbol{\epsilon}^{l m}(k)=\boldsymbol{\epsilon}_{T}(k)\left[\delta^{l m}-k^{l} k^{m} / \mathbf{k}^{2}\right)\right]+\boldsymbol{\epsilon}_{L}(k) k^{l} k^{m} / \mathbf{k}^{2},
$$

and the dispersion equation (32) splits into two equations

$$
\epsilon_{T}(k)=\mathbf{k}^{2} / \omega^{2}, \quad \epsilon_{L}(k)=0 .
$$


The permittivity tensor (33) was calculated for the strongly elongated parton momentum distribution $f(\mathbf{p})$ and it was found $[4,5]$ that there are unstable solutions of the dispersion equation (32).

\section{B. Quark dispersion equation}

The quark dispersion relations are determined by the poles of the hard loop resummed quark propagator or equivalently are found as solutions of the equation

$$
\operatorname{det}[p-\Sigma(p)]=0 .
$$

One sees in Eq. (29) that the spinor structure of $\Sigma$ is very simple: $\Sigma(p)=\gamma^{\mu} \Sigma_{\mu}(p)$. However, we also include here the scalar part which is relevant for the massive quarks. Then,

$$
\Sigma(p)=\gamma^{\mu} \Sigma_{\mu}(p)+C(p) .
$$

Substituting the expression (35) into Eq. (34) and computing the determinant as explained in Appendix 1 of [23], we get

$$
\left[\left(p^{\mu}-\Sigma^{\mu}(p)\right)\left(p_{\mu}-\Sigma_{\mu}(p)\right)-C^{2}(p)\right]^{2}=0 .
$$

When the momentum distribution is isotropic, the structure of $\Sigma$ further simplifies [22]:

$$
\Sigma(p)=A(p) p_{0} \gamma^{0}+B(p) \mathbf{p} \cdot \boldsymbol{\gamma}+C(p) .
$$

Then, the dispersion equation reads

$$
(1-A(p))^{2} p_{0}^{2}-(1-B(p))^{2} \mathbf{p}^{2}-C^{2}(p)=0 .
$$

\section{DISCUSSION}

In the present work we have considered an anisotropic relativistic plasma which is either in equilibrium and the anisotropy is caused by external fields or the plasma is out of equilibrium. In the first case we deal with the homogeneous and static systems while in the second one it can be treated as quasihomogenous and quasistatic for sufficiently short space-time intervals. An example of the first case is the magnetized plasma, while of the second one the parton system from the early stage of relativistic heavy-ion collisions where we encounter a strong anisotropy in the momentum distribution.

The QCD polarization tensor has been computed in two ways. We have first applied the linear-response method within the semiclassical transport theory and then the diagrammatic hard loop approach. The two methods are equivalent (but the distribution functions have to possess a reflection symmetry, i.e., $f(-\mathbf{p})=f(\mathbf{p})$. When using the diagrammatic approach we have referred to the real time formalism since the systems under consideration are, in general, out of equilibrium. According to the hard loop approximation, we have used bare propagators for the internal lines of the polarization tensor which exhibit an explicit aniso- tropic momentum distribution. Another method has been used in $[17,24]$ to study anisotropic relativistic QED plasmas in a strong magnetic field. The system has been assumed there to be in equilibrium and dressed propagators corresponding to electrons in Landau levels have been adopted. In this way, anisotropic distributions arise although the distribution functions depend only on the energy.

As already mentioned, the semiclassical kinetic theory of quarks and gluons has been shown so far to be fully consistent with the QCD dynamics only for quasiequilibrium $[8,9]$. The considerations presented here demonstrate that the equivalence holds for the systems which are far from equilibrium although the space-time homogeneity must be invoked. Thus, the reliability of the kinetic theory methods is improved and in particular, the stability analysis of the parton system form the early stage of ultrarelativistic heavy-ion collisions, which has been based on the linearized kinetic equations, $[4,5]$ is substantiated.

The main advantage of the diagrammatic approach over the transport one is that it allows for a systematic perturbative extension to higher-order effects. Also the fermion selfenergy for anisotropic systems can be calculated in this way. Having the QCD polarization tensor and quark self-energy derived here, one can construct effective gluon and quark propagators from the Dyson-Schwinger equation.

The poles of the effective propagators determine [via Eqs. (32), (36)] the parton dispersion relations in an anisotropic quark-gluon plasma. In the isotropic plasma, the dispersion relations for gluons and quarks show two branches and start from the same energy at zero momentum [21]. The point is that in this case there is no direction preferred and the longitudinal and transverse components of the dielectric function are identical when the momentum vanishes. For the anisotropic systems with a preferred direction even at zero momentum, we expect additional branches and the degeneracy at zero momentum to be removed. In equilibrium all modes are stable or damped due to the Landau mechanism. In the case of anisotropic systems growing modes, i.e., instabilities, are possible. The unstable modes were argued to occur in the parton system from the early stage of ultrarelativistic heavy-ion collisions $[4,5]$. Since the characteristic time of instability development was estimated to be rather small (below $1 \mathrm{fm} / c$ ) these instabilities can significantly influence the temporal evolution of the parton system.

Also the quark dispersion relations following from the effective quark propagator are of physical relevance, as they lead in equilibrium to interesting structures, e.g., van Hove peaks, in the dilepton production rate [25], which might serve as a signature for the quark-gluon plasma formation. It has to be seen whether these structures also survive in the nonequilibrium case.

When the plasma is in the (isotropic) equilibrium state the zero-frequency limit of the longitudinal component of the polarization tensor in the hard thermal loop limit $\left(\Pi_{L}\right.$ $=\Pi_{00}$ ), which is identified with the lowest order Debye screening mass, is finite. The transverse component $\left[\Pi_{T}\right.$ $\left.=\left(\delta_{l m}-k_{l} k_{m} /|\mathbf{k}|^{2}\right) \Pi_{l m} / 2\right]$, on the other hand, shows no static magnetic screening. The situation is much more complicated in the anisotropic plasma. The screening length de- 
pends on the orientation of the vector $\mathbf{k}$ [5], see also [26].

The diagrammatic approach, following the hard loop resummation technique $[10,15]$, allows for a systematic calculation of observables, such as the energy loss of energetic partons or the production of photons and dileptons [18]. Now, the program can be extended to the anisotropic quarkgluon plasma although one has to choose a specific form of the parton momentum distribution. We leave all these issues for future investigations. Here we have intended to provide only the general formalism to study anisotropic systems of quantum fields.

\section{ACKNOWLEDGMENTS}

We are very grateful to the National Institute of Nuclear Theory at Seattle where this project was initiated during the Workshop Non-equilibrium Dynamics in Quantum Field Theory.
[1] Ya. B. Zeldovich, A.A. Ruzmaikin, and D.D. Sokoloff, Magnetic Fields in Astrophysics (Gordon and Breach, New York, 1983); G.G. Raffelt, Stars as Laboratories for Fundamental Physics: The Astrophysics of Neutrinos, Axions, and other Weakly Interacting Particles (University of Chicago Press, Chicago, 1996).

[2] K. Geiger, Phys. Rep. 258, 237 (1995).

[3] X.-N. Wang, Phys. Rep. 280, 287 (1997).

[4] St. Mrówczyński, Phys. Lett. B 314, 118 (1993); 393, 26 (1997).

[5] St. Mrówczyński, Phys. Rev. C 49, 2191 (1994).

[6] H.-Th. Elze and U. Heinz, Phys. Rep. 183, 81 (1989).

[7] St. Mrówczyński, Phys. Rev. D 39, 1940 (1989).

[8] J.-P. Blaizot and E. Iancu, Nucl. Phys. B417, 608 (1994).

[9] J.-P. Blaizot and E. Iancu, Nucl. Phys. B557, 183 (1999).

[10] E. Braaten and R.D. Pisarski, Nucl. Phys. B337, 569 (1990).

[11] P.F. Kelly, Q. Liu, C. Lucchesi, and C. Manuel, Phys. Rev. D 50, 4209 (1994).

[12] T.S. Biró, E. van Doorn, B. Müller, M.H. Thoma, and X.-N. Wang, Phys. Rev. C 48, 1275 (1993).

[13] R. Baier, M. Dirks, K. Redlich, and D. Schiff, Phys. Rev. D 56, 2548 (1997).

[14] M. Le Bellac and H. Mabilat, Z. Phys. C 75, 137 (1997).
[15] M.E. Carrington, H. Defu, and M.H. Thoma, Eur. Phys. J. C 7, 347 (1999).

[16] R.D. Pisarski, hep-ph/9710370.

[17] P. Elmfors, Nucl. Phys. B487, 207 (1997).

[18] M.H. Thoma, in Quark-Gluon Plasma 2, edited by R. Hwa (World Scientific, Singapore, 1995), p.51.

[19] K. Chou, Z. Su, B. Hao, and L. Yu, Phys. Rep. 145, 1 (1985).

[20] A. Peshier, K. Schertler, and M.H. Thoma, Ann. Phys. (N.Y.) 266, 162 (1998).

[21] V.V. Klimov, Zh. Eksp. Teor. Fiz. 82, 336 (1982) [Sov. Phys. JETP 55, 199 (1982)]; H.A. Weldon, Phys. Rev. D 26, 1394 (1982).

[22] H.A. Weldon, Phys. Rev. D 26, 2789 (1982); J.-P. Blaizot and J.-Y. Ollitrault, 48, 1390 (1993).

[23] St. Mrówczyński and U. Heinz, Ann. Phys. (N.Y.) 229, 1 (1994).

[24] M.P. Kennett and D.B. Melrose, Phys. Rev. D 58, 093011 (1998).

[25] E. Braaten, R.D. Pisarski, and T.C. Yuan, Phys. Rev. Lett. 64, 2242 (1990); A. Peshier and M.H. Thoma, ibid. 84, 891 (2000).

[26] T.S. Biró, B. Müller, and X.-N. Wang, Phys. Lett. B 283, 171 (1992). 